\title{
ON THE $2^{n}-1$ LATTICE OF THE COLLATZ CONJECTURE.
}

\author{
Alex Nguhi ${ }^{1} 1$
}

\begin{abstract}
The elements of $2^{n}-1$ when arranged in ascending order of $n, n \in \mathbf{N}$ form a fine lattice with respect to some diagonal. The focus of this paper is on the leading diagonal in the pursuit of reducing the calculational complexity. One such short cut will focus on the summands of the powers of 3. The finding is that $\sum_{i=0}^{n} 3^{i}$ exhibits a Collatz like behavior. Armed with that we can reduce the amount of cycles considerably.
\end{abstract}

\section{INTRODUCTION}

This paper explores the findings of a previous paper on Dynamical Systems of the form $\delta k+\beta$ systems, $\delta, \beta \in \mathbf{N}$ for more info [1]. The area of study is on the sequence of odd numbers.

\section{THE COLLATZ PROBLEM REVISITED}

\subsection{Lattices of $2^{n}-1$}

Let's define a set $\mathbf{S}$ such that $\mathbf{S}=2^{n}-1 ; n \in \mathbf{N}$. The idea is finding the next odd number from the operation $3 \mathrm{k}+1, k \in \mathbf{S}$. The Collatz Conjecture is also called the $3 \mathrm{x}+1$ problem in mathematical literature, [2]

Therefore applying a fuction, $f$ that defines the Collatz problem implies finding the next odd number

Example

$f(3)=\frac{3 \times 3+1}{2^{1}}=5$

Hence, $f(k)=\frac{3 \times k+1}{2^{c}}, c \in \mathbf{N}$

Below are some sequences of the lattice described above:

$$
\begin{aligned}
& 1=1 \\
& 3=5,1 \\
& 7=11,17,13,5,1 \\
& 15=23,35,53,5,1 \\
& 31=47,71,107,161,121,91, \ldots, 325,61,23,35,53,5,1 \\
& 63=95,143,215,323,485,91, \ldots, 325,61,23,35,53,5,1
\end{aligned}
$$

This was previously defined as a chaotic matrix due to its behaviour.

\footnotetext{
1

alexnguhi@gmail.com
} 


\begin{tabular}{|c|c|c|c|c|c|c|c|c|c|c|c|c|c|c|}
\hline 1 & 1 & 1 & 1 & 1 & 1 & 1 & 1 & 1 & 1 & 1 & 1 & 1 & 1 & 1 \\
\hline 3 & 5 & 1 & 1 & 1 & 1 & 1 & 1 & 1 & 1 & 1 & 1 & 1 & 1 & 1 \\
\hline 7 & 11 & 17 & 13 & 5 & 1 & 1 & 1 & 1 & 1 & 1 & 1 & 1 & 1 & 1 \\
\hline 15 & 23 & 35 & 53 & 5 & 1 & 1 & 1 & 1 & 1 & 1 & 1 & 1 & 1 & 1 \\
\hline 31 & 47 & 71 & 107 & 161 & 121 & 91 & $\ldots$ & 325 & 61 & 23 & 35 & 53 & 5 & 1 \\
\hline 63 & 95 & 143 & 215 & 323 & 485 & 91 & $\ldots$ & 325 & 61 & 23 & 35 & 53 & 5 & 1 \\
\hline. & & & & & & & & & & & & & & \\
\hline .. & & & & & & & & & & & & & & \\
\hline$k$ & $\ldots$ & $\ldots$ & $\ldots$ & ... & ... & $\ldots$ & $\alpha_{n-1}$ & $\ldots$ &.. & $\ldots$ & & & & \\
\hline $2 k+1$ & $\ldots$ & $\ldots$ & $\ldots$ & $\ldots$ & $\ldots$ & $\ldots$ & $\ldots$ & $\alpha_{n}$ & $\ldots$ & $\ldots$ & $\ldots$ & & & \\
\hline
\end{tabular}

There's no limit to the number of columns because when we get to 1 , $f(1)=1$. The leading diagonal is in bold and perhaps may be denoted by $\alpha_{i}$

Previously it was established :

$\alpha_{0}=1$

$\alpha_{1}=3 \times \alpha_{0}+2=5$

$\alpha_{2}=3 \times \alpha_{1}+2=17$

$\cdots$

$\cdots$

$\alpha_{n}=3 \times \alpha_{n-1}+2$

\subsection{A numerical Analysis of the Diagonals}

A lot of difficulty emerges in deriving the diagonal entry for large $\mathrm{n}$ of $2^{n}-1$ . In the old fashioned way, it's done through brute force. But mathematically, we can derive a formula for such purposes.

Rewriting gives,

$\alpha_{0}=1$

$\alpha_{1}=(3 \times 1+2)=5$

$\alpha_{2}=((3 \times 1+2) 3+2)=17$

$\alpha_{3}=(((3 \times 1+2) 3+2) 3+2)=53$

$\begin{array}{llllll}\cdots & \cdots & \cdots & \cdots\end{array}$

$\cdots$

.

$\alpha_{n}=(\ldots((((3 \times 1+2) 3+2) 3+2) 3+2) 3 \ldots+. .2)$

The idea of closed forms introduced previously is visible from that inductive formula.

An expanded formuale 


$$
\begin{aligned}
& \alpha_{0}=1 \\
& \alpha_{1}=3.1+2=5 \\
& \alpha_{2}=3^{2}+3.2+2=17 \\
& \alpha_{3}=3^{3}+3^{2} .2+3.2+2=53 \\
& \alpha_{4}=3^{4}+3^{3} .2+3^{2} .2+3.2+2=161 \\
& \cdots \\
& \quad \ldots \\
& \cdots \\
& \alpha_{n}=3^{n}+\ldots+3^{3} .2+3^{2} .2+3.2+2
\end{aligned}
$$

This is clearly a binomial problem but mostly importantly it involves the summation of powers of 3 .

\section{Lemma 1.1}

The general formuala of the n'th diagonal element in the chaotic matrix is given by :-

$$
\alpha_{n}=3^{n}+2\left(\sum_{i=0}^{n-1} 3^{i}\right)
$$

This deceptively simple formula is still too 'general' therefore needing a more accurate version. Later propositions will iron out this.

\section{Lemma 2.2}

The series derived from the sumands of $\sum_{i=0}^{n} 3^{i}$ are succesive $3 x+1$ problems.

Proof

\section{Observe:}

$$
\begin{aligned}
& 1 \\
& 3 \times 1+1=3^{0}+3^{1}=4 \\
& 3 \times 4+1=3^{0}+3^{1}+3^{2}=13 \\
& 3 \times 13+1=3^{0}+3^{1}+3^{2}+3^{3}=40 \\
& 3 \times 40+1=3^{0}+3^{1}+3^{2}+3^{3}+3^{4}=121 \\
& \ldots \\
& \quad \ldots \\
& \cdots \\
& \sum_{i=0}^{n-1} 3^{i}=z \\
& 3 \times z+1=\sum_{i=0}^{n} 3^{i}
\end{aligned}
$$


By mathematical induction Lemma 1.2 is true

\section{Corollary 1.1}

The sum of the powers of 3 can be summarized by :-

$$
\sum_{i=0}^{n} 3^{i}=3^{n}+\frac{3^{n}-1}{2}
$$

\section{Lemma 1.3}

The n'th diagonal element is given by the formulae :

$$
\alpha_{n}=2\left(3^{n}\right)-1
$$

Proof

The summation of $\mathrm{n}$ powers of 3 can be simplified further.

Let $\mathrm{z}=\sum_{i=0}^{n-1} 3^{i}$

$3^{n}=2 z+1$

$z=\frac{3^{n}-1}{2}$

Using Mathematical Induction.

$$
\begin{aligned}
& 1 \\
& 3+(3-1) / 2=4 \\
& 3^{2}+(9-1) / 2=13 \\
& 3^{3}+(27-1) / 2=40 \ldots \\
& \quad \ldots \\
& \ldots \\
& \sum_{i=0}^{n} 3^{i}=3^{n}+\left(3^{n}-1\right) / 2
\end{aligned}
$$

Since

$$
\alpha_{n}=3^{n}+2\left(\sum_{i=0}^{n-1} 3^{i}\right)
$$

Substitute z for $3^{n}$

$$
\begin{gathered}
=3^{n}+2 z=2 z+1+2 z \\
=2\left(\frac{3^{n}-1}{2}\right)+2\left(\frac{3^{n}-1}{2}\right)+1 \\
4\left(\frac{3^{n}}{2}\right)-2+1 \\
=2.3^{n}-1
\end{gathered}
$$


If that's the case for sums, we can apply Corollary 1 . Hence, lemma 1.3 is just a pithy extension that doesn't involve tiresome sums.

\section{Corollary 1.2 - Fibonacci-Like Sums}

Lemma 1.3 means that the $\alpha_{i}^{\prime} s$ are succesive Fibonacci-like sums.

Observe :

$$
\begin{aligned}
& \sum_{i=0}^{n} 3^{i}=1,4,13,40,121,364, \ldots \\
& \text { Diagonals }=1,5,17,53,161,485, \ldots
\end{aligned}
$$

So : Let $\mathbf{M}$ denote the set of the sums of the powers of 3 ie $\mathbf{M}=1,4,13,40, \ldots$ and $m_{i} \in \mathbf{M}$ then:

$$
\begin{gathered}
\alpha_{n}=m_{n}+m_{n-1} \\
=3^{n}+\frac{3^{n}-1}{2}+3^{n-1}+\frac{3^{n-1}-1}{2} \\
\frac{2.3^{n}+3^{n}-1}{2}+\frac{2.3^{n-1}+3^{n-1}-1}{2} \\
\frac{3^{n+1}+3^{n}-2}{2}
\end{gathered}
$$

\section{APPLICATIONS.}

Find the values of $\alpha_{i}$ :

a. for $\alpha_{3}$

Simply $\alpha_{3}=2\left(3^{i}\right)-1$

$$
2 \times 27-1=53
$$

OR

$$
\begin{aligned}
& \mathrm{z}=\sum_{i=0}^{3-1} 3^{i}=13 \\
& \text { with } \sum_{i=0}^{3} 3^{i}=3^{3}+13=40 \\
& \text { hence }
\end{aligned}
$$

$$
\alpha_{3}=13+40=53
$$

Alternatively,

Using $\alpha_{n}=\frac{3^{n+1}+3^{n}-2}{2}$ 


$$
\alpha_{3}=\frac{3^{3+1}+3^{3}-2}{2}
$$

$$
\alpha_{3}=\frac{81+27-2}{2}=53
$$

b. $\alpha_{30}$

$$
\alpha_{30}=2\left(3^{30}\right)-1=411,782,264,189,297
$$

\subsection{SYNCHRONIZATION AND DIAGONALS.}

Previously it was asserted that synchronization occurs somewhere along the rows but only after the system has attained its diagonal entry .

Examples of Collatz sequences for various $\alpha_{i}$

1. $\alpha_{30}=411,782,264,189,297$

This is most likely to be synchronized with $\alpha_{31}$ as shown from this result of a simple program. The elements of both sets synchronize right away(have similar collatz sequences)

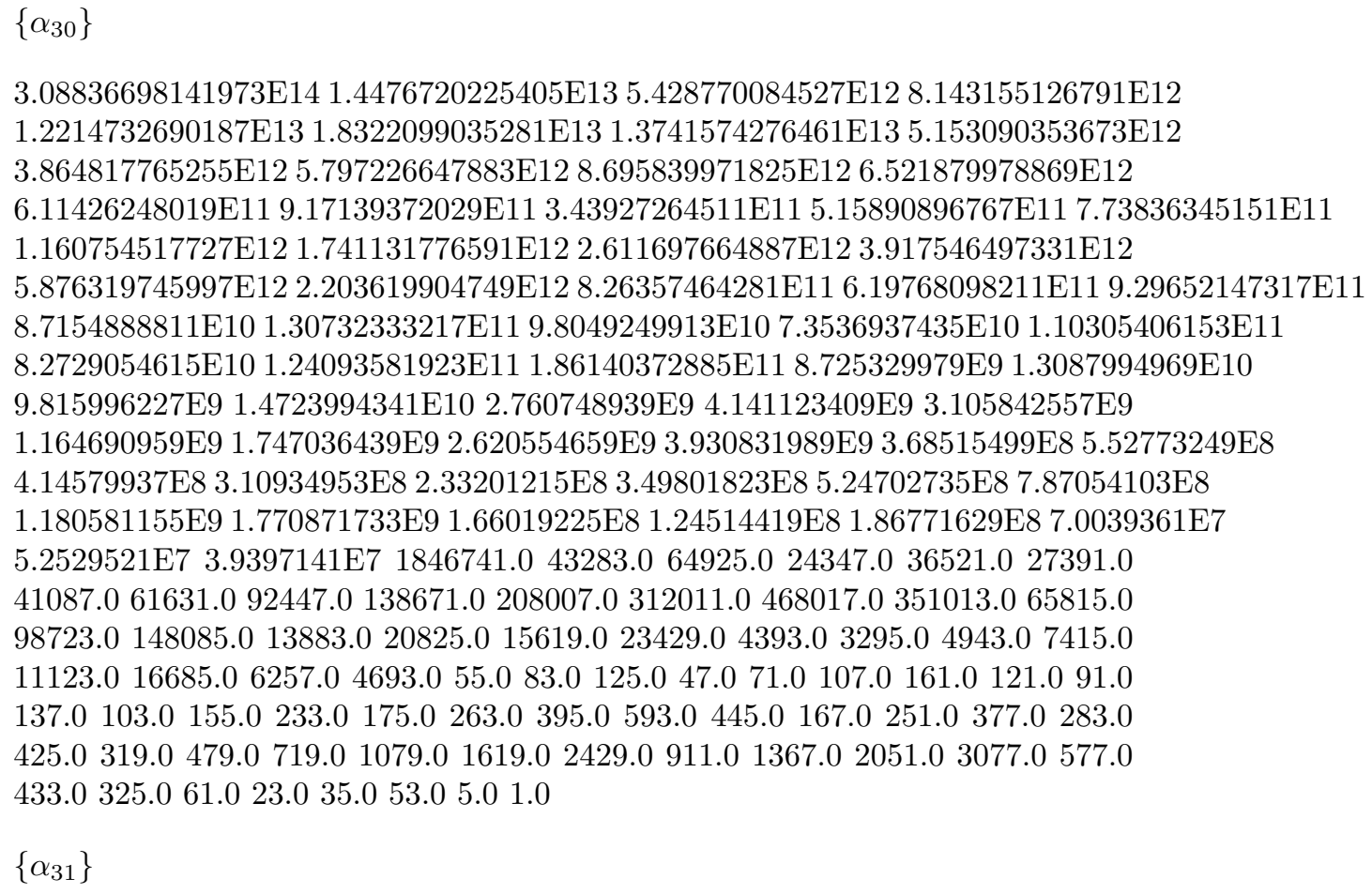


1.4476720225405E13 5.428770084527E12 8.143155126791E12 1.2214732690187E13 1.8322099035281E13 1.3741574276461E13 5.153090353673E12 3.864817765255E12 5.797226647883E12 8.695839971825E12 6.521879978869E12 6.11426248019E11 9.17139372029E11 3.43927264511E11 5.15890896767E11 7.73836345151E11 1.160754517727E12 1.741131776591E12 2.611697664887E12 3.917546497331E12 5.876319745997E12 2.203619904749E12 8.26357464281E11 6.19768098211E11 9.29652147317E11 8.7154888811E10 1.30732333217E11 9.8049249913E10 7.3536937435E10 1.10305406153E11 8.2729054615E10 1.24093581923E11 1.86140372885E11 8.725329979E9 1.3087994969E10 9.815996227E9 1.4723994341E10 2.760748939E9 4.141123409E9 3.105842557E9 1.164690959E9 1.747036439E9 2.620554659E9 3.930831989E9 3.68515499E8 5.52773249E8 4.14579937E8 3.10934953E8 2.33201215E8 3.49801823E8 5.24702735E8 7.87054103E8 1.180581155E9 1.770871733E9 1.66019225E8 1.24514419E8 1.86771629E8 7.0039361E7 5.2529521E7 3.9397141E7 1846741.0 43283.0 64925.0 24347.0 36521.0 27391.0 41087.0 61631.0 92447.0138671 .0208007 .0312011 .0468017 .0351013 .065815 .098723 .0148085 .0

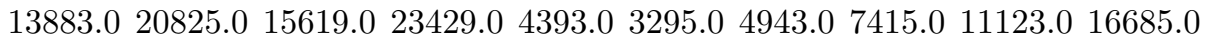
6257.04693 .055 .083 .0125 .047 .071 .0107 .0161 .0121 .091 .0137 .0103 .0155 .0 $233.0 \quad 175.0263 .0 \quad 395.0 \quad 593.0 \quad 445.0 \quad 167.0 \quad 251.0 \quad 377.0 \quad 283.0 \quad 425.0 \quad 319.0479 .0$

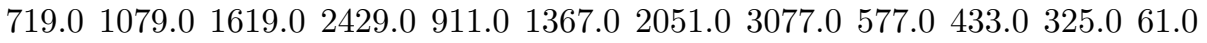
23.035 .053 .05 .01 .0

[Program finished]

2. $\left\{\alpha_{100}\right\}$ and $\left\{\alpha_{101}\right\}$

$\left\{\alpha_{100}\right\}$

3.176259199493869E15 1.191097199810201E15 8.93322899857651E14 1.339984349786477E15 5.02494131169929E14 3.76870598377447E14 5.65305897566171E14 8.47958846349257E14 6.35969134761943E14 9.53953702142915E14 1.430930553214373E15 2.68299478727695E14 4.02449218091543E14 6.03673827137315E14 9.05510740705973E14 8.4891631941185E13 6.3668723955889E13 4.7751542966917E13 8.953414306297E12 6.715060729723E12 1.0072591094585E13 7.554443320939E12 1.1331664981409E13 8.498748736057E12 6.374061552043E12 9.561092328065E12 7.170819246049E12 5.378114434537E12 4.033585825903E12 6.050378738855E12 9.075568108283E12 1.3613352162425E13 1.0210014121819E13 1.5315021182729E13 1.1486265887047E13 1.7229398830571E13 $2.5844098245857 \mathrm{E} 13$ 1.9383073684393E13 1.4537305263295E13 2.1805957894943E13 3.2708936842415E13 4.9063405263623E13 7.3595107895435E13 1.10392661843153E14 8.2794496382365E13 3.1047936143387E13 4.6571904215081E13 3.4928928161311E13 5.2393392241967E13 7.8590088362951E13 1.17885132544427E14 1.76827698816641E14 $1.32620774112481 \mathrm{E} 14$ 9.9465580584361E13 7.4599185438271E13 1.11898778157407E14 $1.67848167236111 \mathrm{E} 14$ 2.51772250854167E14 3.77658376281251E14 5.66487564421877E14 $5.3108209164551 \mathrm{E} 13$ 7.9662313746827E13 1.19493470620241E14 8.9620102965181E13 3.3607538611943E13 5.0411307917915E13 7.5616961876873E13 5.6712721407655E13 8.5069082111483E13 1.27603623167225E14 9.5702717375419E13 1.43554076063129E14 1.07665557047347E14 1.61498335571021E14 6.0561875839133E13 2.2710703439675E13 3.4066055159513E13 2.5549541369635E13 3.8324312054453E13 3.592904255105E12 2.694678191329E12 2.021008643497E12 1.515756482623E12 2.273634723935E12 $3.410452085903 \mathrm{E} 12$ 5.115678128855E12 7.673517193283E12 1.1510275789925E13 
2.158176710611E12 3.237265065917E12 1.213974399719E12 1.820961599579E12 2.731442399369E12 2.048581799527E12 3.072872699291E12 4.609309048937E12 3.456981786703E12 5.185472680055E12 7.778209020083E12 1.1667313530125E13 4.375242573797E12 8.20357982587E11 1.230536973881E12 9.22902730411E11 1.384354095617E12 1.038265571713E12 7.78699178785E11 5.84024384089E11 4.38018288067E11 6.57027432101E11 1.23192643519E11 1.84788965279E11 2.77183447919E11 4.15775171879E11 6.23662757819E11 9.35494136729E11 7.01620602547E11 1.052430903821E12 3.94661588933E11 7.3999047925E10 6.937410743E9 1.0406116115E10 1.5609174173E10 5.853440315E9 8.780160473E9 6.585120355E9 9.877680533E9 4.63016275E8 6.94524413E8 2.60446655E8 3.90669983E8 5.86004975E8 8.79007463E8 1.318511195E9 1.977766793E9 1.483325095E9 2.224987643E9 3.337481465E9 2.503111099E9 3.754666649E9 2.815999987E9 4.223999981E9 1.583999993E9 1.187999995E9 1.781999993E9 1.336499995E9 2.004749993E9 1.503562495E9 2.255343743E9 3.383015615E9 5.074523423E9 7.611785135E9 1.1417677703E10 1.7126516555E10 2.5689774833E10 1.9267331125E10 1.806312293E9 3.38683555E8 5.08025333E8 4.7627375E7 7.1441063E7 1.07161595E8 1.60742393E8 1.20556795E8 1.80835193E8 1.35626395E8 2.03439593E8 1.52579695E8 2.28869543E8 3.43304315E8 5.14956473E8 3.86217355E8 5.79326033E8 4.34494525E8 1.62935447E8 2.44403171E8 3.66604757E8 8592299.0 1.2888449E7 9666337.0 7249753.0 5437315.0 8155973.0 1529245.0 573467.0 860201.0645151 .0967727 .01451591 .02177387 .03266081 .02449561 .01837171 .0

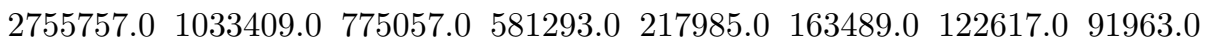
137945.0103459 .0155189 .014549 .0341 .01 .0

$\left\{\alpha_{101}\right\}$

1.191097199810201E15 8.93322899857651E14 1.339984349786477E15 5.02494131169929E14 3.76870598377447E14 5.65305897566171E14 8.47958846349257E14 6.35969134761943E14 9.53953702142915E14 1.430930553214373E15 2.68299478727695E14 4.02449218091543E14 6.03673827137315E14 9.05510740705973E14 8.4891631941185E13 6.3668723955889E13 $4.7751542966917 \mathrm{E} 13$ 8.953414306297E12 6.715060729723E12 1.0072591094585E13 7.554443320939E12 1.1331664981409E13 8.498748736057E12 6.374061552043E12 $9.561092328065 \mathrm{E} 12$ 7.170819246049E12 5.378114434537E12 4.033585825903E12 $6.050378738855 \mathrm{E} 12$ 9.075568108283E12 1.3613352162425E13 1.0210014121819E13 $1.5315021182729 \mathrm{E} 131.1486265887047 \mathrm{E} 13$ 1.7229398830571E13 2.5844098245857E13 1.9383073684393E13 1.4537305263295E13 2.1805957894943E13 3.2708936842415E13 4.9063405263623E13 7.3595107895435E13 1.10392661843153E14 8.2794496382365E13 3.1047936143387E13 4.6571904215081E13 3.4928928161311E13 5.2393392241967E13 7.8590088362951E13 1.17885132544427E14 1.76827698816641E14 1.32620774112481E14 $9.9465580584361 \mathrm{E} 13$ 7.4599185438271E13 1.11898778157407E14 1.67848167236111E14 2.51772250854167E14 3.77658376281251E14 5.66487564421877E14 5.3108209164551E13 7.9662313746827E13 1.19493470620241E14 8.9620102965181E13 3.3607538611943E13 $5.0411307917915 \mathrm{E} 13$ 7.5616961876873E13 5.6712721407655E13 8.5069082111483E13 $1.27603623167225 \mathrm{E} 14$ 9.5702717375419E13 1.43554076063129E14 1.07665557047347E14 1.61498335571021E14 6.0561875839133E13 2.2710703439675E13 3.4066055159513E13 $2.5549541369635 \mathrm{E} 13$ 3.8324312054453E13 3.592904255105E12 2.694678191329E12 2.021008643497E12 1.515756482623E12 2.273634723935E12 3.410452085903E12 $5.115678128855 \mathrm{E} 12$ 7.673517193283E12 1.1510275789925E13 2.158176710611E12 
3.237265065917E12 1.213974399719E12 1.820961599579E12 2.731442399369E12 2.048581799527E12 3.072872699291E12 4.609309048937E12 3.456981786703E12 5.185472680055E12 7.778209020083E12 1.1667313530125E13 4.375242573797E12 8.20357982587E11 1.230536973881E12 9.22902730411E11 1.384354095617E12 1.038265571713E12 7.78699178785E11 5.84024384089E11 4.38018288067E11 6.57027432101E11 1.23192643519E11 $1.84788965279 \mathrm{E} 11$ 2.77183447919E11 4.15775171879E11 6.23662757819E11 9.35494136729E11 7.01620602547E11 1.052430903821E12 3.94661588933E11 7.3999047925E10 6.937410743E9 1.0406116115E10 1.5609174173E10 5.853440315E9 8.780160473E9 6.585120355E9 9.877680533E9 4.63016275E8 6.94524413E8 2.60446655E8 3.90669983E8 5.86004975E8 8.79007463E8 1.318511195E9 1.977766793E9 1.483325095E9 2.224987643E9 3.337481465E9 2.503111099E9 3.754666649E9 2.815999987E9 4.223999981E9 1.583999993E9 1.187999995E9 1.781999993E9 1.336499995E9 2.004749993E9 1.503562495E9 2.255343743E9 3.383015615E9 5.074523423E9 7.611785135E9 1.1417677703E10 1.7126516555E10 2.5689774833E10 1.9267331125E10 1.806312293E9 3.38683555E8 5.08025333E8 4.7627375E7 7.1441063E7 $1.07161595 \mathrm{E} 81.60742393 \mathrm{E} 81.20556795 \mathrm{E} 81.80835193 \mathrm{E} 81.35626395 \mathrm{E} 82.03439593 \mathrm{E} 8$ 1.52579695E8 2.28869543E8 3.43304315E8 5.14956473E8 3.86217355E8 5.79326033E8 4.34494525E8 1.62935447E8 2.44403171E8 3.66604757E8 8592299.0 1.2888449E7 9666337.07249753 .05437315 .08155973 .01529245 .0573467 .0860201 .0645151 .0 967727.01451591 .02177387 .03266081 .02449561 .01837171 .02755757 .01033409 .0 775057.0581293 .0217985 .0163489 .0122617 .091963 .0137945 .0103459 .0155189 .0 14549.0341 .01 .0

[Program finished]

\section{BIBLIOGRAPHY}

1. A,Nguhi \& C, Kweyu(2021). On Generalized Convergence in Collatz Systems.

2. Andrei, S., \& Masalagiu, C. (1998). About the Collatz conjecture. Acta Informatica, 35(2), 167-179 